\title{
Coeliac disease with histological features of peptic duodenitis: Value of assessment of intraepithelial lymphocytes
}

\author{
M D Jeffers, D O'B Hourihane
}

\begin{abstract}
Aims-To determine if a clinically important polymorphonuclear leucocyte infiltrate and surface gastric epithelial metaplasia occur in the second part of the duodenum in coeliac disease; to evaluate the utility of these morphological criteria in the differential diagnosis of coeliac disease and peptic duodenitis.

Methods -49 mucosal biopsy specimins of the second part of the duodenum reported as showing inflammation were reviewed. Sections were prepared with haematoxylin and eosin, periodic acid Schiff, and Warthin-Starry stains. Clinical presentation, outcome, and immunological investigations were assessed.

Results-Four cases confirmed as coeliac disease on clinical and immunological grounds showed acute inflammation and surface epithelial gastric metaplasia. Increased intraepithelial lymphocytes (IELs) were found in each of the four. Conclusions-Clinically important polymorphonuclear leucocyte infiltration and surface epithelial gastric metaplasia may occur in the duodenal mucosa in coeliac disease and should not be used as diagnostic features to exclude the diagnosis of coeliac disease in the absence of confirmatory clinical and immunological information.
\end{abstract}

(F Clin Pathol 1993;46:420-424)

Inflammation of the duodenal mucosa occurs in many different diseases. Specific morphological features are present which point to aetiology in some cases, while the remainder are often grouped together under the collective title of non-specific (peptic) duodenitis. Coeliac disease is characterised on mucosal biopsy specimens by villous atrophy, surface epithelial cell disarray, mixed chronic inflammatory cell infiltrate of the lamina propria, and a sharp increase in intraepithelial lymphocytes, chiefly of the T8 + subtype. In peptic duodenitis the inflammation includes prominent foci of polymorphonuclear leucocytes, and flattening of surface (villous atrophy), and may be difficult to distinguish from the changes seen in coeliac disease. Features which have been proposed as useful diagnostic pointers in distinguishing severe peptic duodenitis from coeliac disease include the relative lack of architectural changes in nonspecific duodenitis, and the scanty polymor- phonuclear leucocyte infiltrate and absence of gastric metaplasia in coeliac disease.

We present four cases in which mucosal biopsy specimens of the second part of the duodenum presented histological appearances suggesting non-specific duodenitis but in which the diagnosis of coeliac disease was subsequently confirmed on clinical, immunological, and histological grounds.

\section{Methods}

All mucosal biopsy specimens of the second part of the duodenum reported as showing inflammation were reviewed for the 6 months from January to July 1991. This included cases reported as coeliac disease, peptic duodenitis, non-specific inflammation and other specific diseases. Sections $4 \mu \mathrm{m}$ thick were prepared with haematoxylin and eosin, periodic acid Schiff (PAS), and Warthin-Starry silver stains. Sections were reviewed with particular emphasis on villous atrophy, crypt hyperplasia, chronic inflammation, intraepithelial lymphocytes, acute inflammation, surface epithelial abnormalities and surface epithelial gastric metaplasia. On the basis of morphology, cases were designated as coeliac disease, peptic duodenitis, non-specific inflammation and other specific inflammatory disorders.

Case notes were reviewed with particular reference to clinical presentation, endoscopic appearance, response to gluten withdrawal and serum $a$ gliadin and endomysial antibody titres. Intraepithelial lymphocytes were counted for 300 surface epithelial cells, and the result expressed as a mean, with the range per 100 surface epithelial cells. Alpha gliadin antibodies are present from $0-3$ units $/ \mathrm{ml}$ in healthy subjects.

\section{Results}

The morphological diagnoses of the 49 biopsy specimens from 48 patients were as follows: coeliac disease $n=13$; peptic duodenitis $n=12$; peptic ulceration $n=3$; non-specific inflammation $n=16$; Crohn's disease $n=1$; coeliac disease/peptic duodenitis $n=4$.

Helicobacter-like organisms were identified in four cases of peptic duodenitis, and were present only on gastric type epithelium.

Serum $a$ gliadin antibody titres were raised in 10 cases (coeliac disease nine cases, range 6 to 30 units $/ \mathrm{ml}$, mean 13.1 units $/ \mathrm{ml}$, Crohn's disease one case, 32 units $/ \mathrm{ml}$ ).

\author{
D O'B Hourihan \\ Correspondence to: \\ Professor D O'B Hourihane \\ Accepted for publication \\ 4 November 1992 \\ Pathology, St James's \\ Hospital and Trini \\ Ireland
}


Serum endomysial antibody titre was available in four cases of coeliac disease and was positive in two cases.

Gastric metaplasia was present in 15 cases, 12 cases of peptic duodenitis, and three cases of coeliac disease originally reported as most suggestive of peptic duodenitis. Biopsy specimens from these three patients showed architectural changes and inflammatory infiltration suggestive of coeliac disease but also contained acute inflammation and surface epithelial gastric metaplasia. These three cases are presented in detail below. A fourth example of gastric metaplasia and focal polymorphonuclear leucocyte exudate was encountered during 1992, and has been added to the series (case 4 ).

\section{Case reports}

CASE 1

A 42 year old man presented with a history of persistent unexplained iron deficiency anaemia (haemoglobin $114 \mathrm{~g} / \mathrm{l}$, mean cell volume $74.5 \mathrm{fl})$. There was no history of diarrhoea, nausea, weight loss, mouth ulcers or

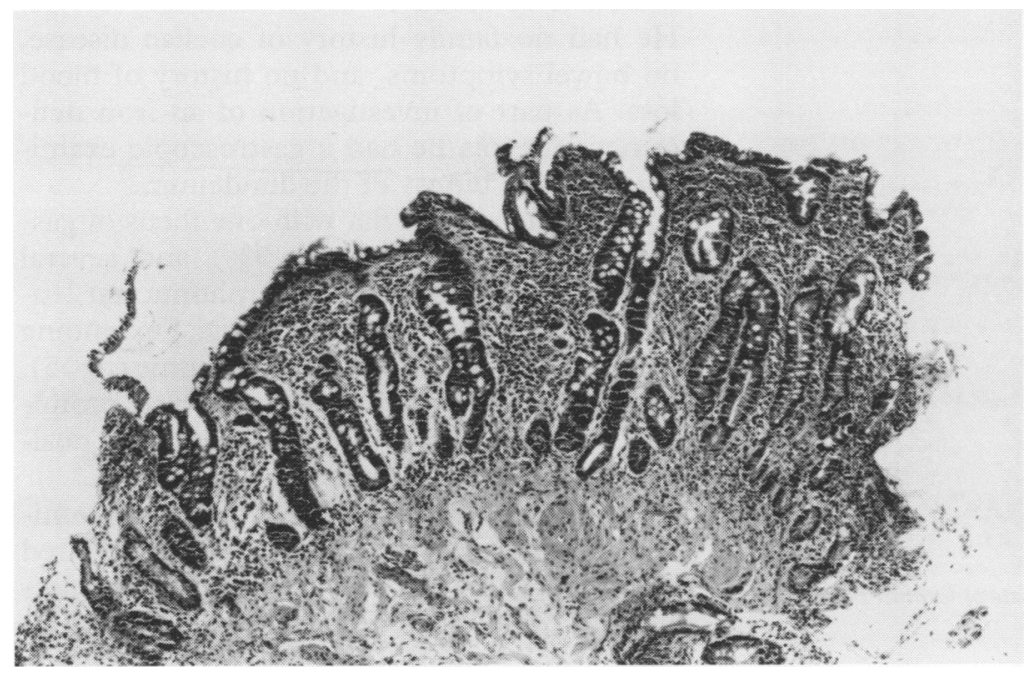

Figure 1 Case 1: duodenal mucosa with villous atrophy and severe inflammmation (haematoxylin and eosin.)

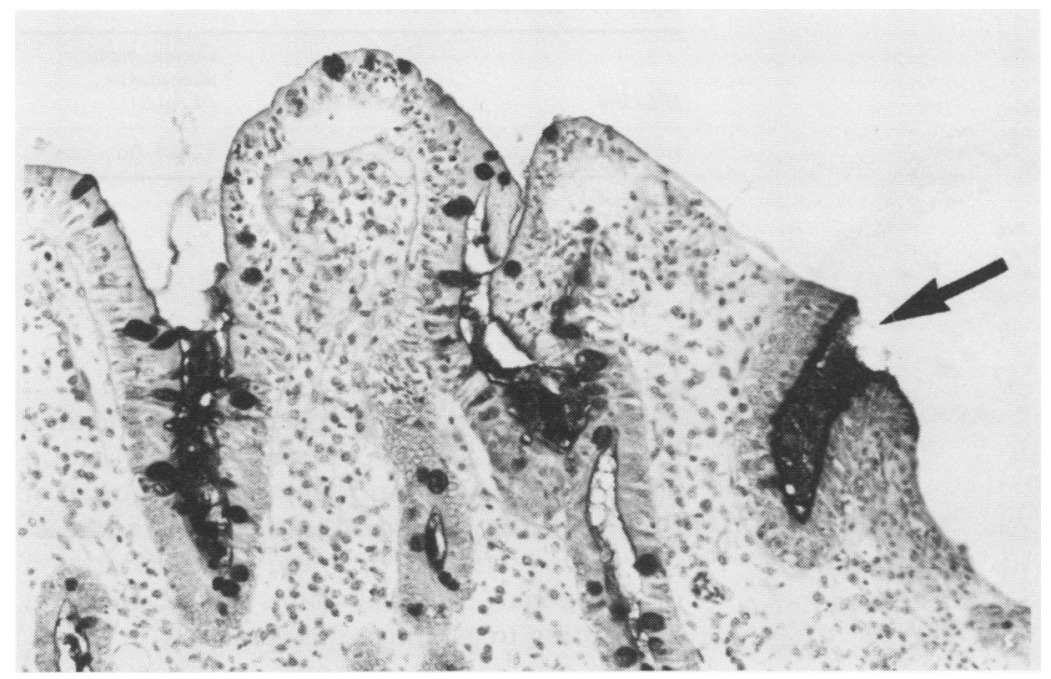

Figure 2 Case 1: focal surface epithelial gastric metaplasia (arrow) (periodic acid-Schiff. other symptoms.

There was no family history of coeliac diseae, although one sibling also had iron deficiency anaemia. At endoscopy the oesophagus, stomach, and duodenum were all macroscopically normal. A biopsy specimen of the second part of the duodenum showed villous atrophy (fig 1 ) and prominent intraepithelial lymphocytes, with focal gastric metaplasia (less than $5 \%$ of surface enterocytes) (fig 2) and pronounced polymorphonuclear leucocyte infiltrate in the lamina propria which extended into the surface epithelium. This was interpreted as peptic duodenitis rather than coeliac disease. Serum $a$ gliadin antibodies were positive at a titre of $>32$ units $/ \mathrm{ml}$ and serum endomysial antibodies (EMA) were positive.

A gluten challenge was performed $(50 \mathrm{~g}$ gluten a day for 10 weeks) and a biopsy specimen of the second part of the duodenum was taken at the end of this. This again showed architectural features suggestive of coeliac disease, but it also showed a noticeable polymorphonuclear leucocyte infiltrate, raising the possibility of non-specific duodenitis; $a$ gliadin antibodies was again positive at a titre of $>32$ units $/ \mathrm{ml}$, and EMA were positive. The patient was started on a gluten free diet. After 6 months a duodenal biopsy was performed which showed that the mucosa had reverted to normal (fig 3).

CASE 2

A 13 year old girl who had not yet begun to menstruate presented with lassitude and fatigue and was found to have iron deficiency anaemia (haemoglobin $37 \mathrm{~g} / \mathrm{l}$, mean cell volume $67.6 \mathrm{fl})$. She was also folate deficient $(1.2 \mathrm{mg} / 1$ normal $2.7>20 \mathrm{mg} / \mathrm{l})$. She had no gastrointestinal symptoms. There was no family history of coeliac disease.

At endoscopy the oesophagus and stomach appeared normal and the duodenum appeared atrophic. A mucosal biopsy specimen from the second part of the duodenum showed villous atrophy, mixed chronic inflammatory infiltrate of the lamina propria, increased intraepithelial lymphocytes, and a polymorphonuclear leucocyte infiltrate of lamina propria, crypt, and surface epithelium (fig 4), and focal gastric metaplasia of surface epithelium. Serum $a$ gliadin antibodies were positive, at a titre of 30 units/ml; EMA were positive.

\section{CASE 3}

A 74 year old woman was admitted with weakness and dyspnoea. Other symptoms included heartburn, anorexia, and a weight loss of $5 \mathrm{lbs}$. A full blood count showed iron deficiency anaemia (haemoglobin $83 \mathrm{~g} / 1$, mean cell volume $82 \mathrm{fl}$ ). At endoscopy there was oesophagitis at $28 \mathrm{~cm}$ and the stomach and duodenum were macroscopically normal. A mucosal biopsy specimen of the oesophagus showed changes suggestive of reflux oesophagitis. A biopsy specimen of the second part of the duodenum showed subtotal villous atrophy with crypt hyperplasia, epithe- 


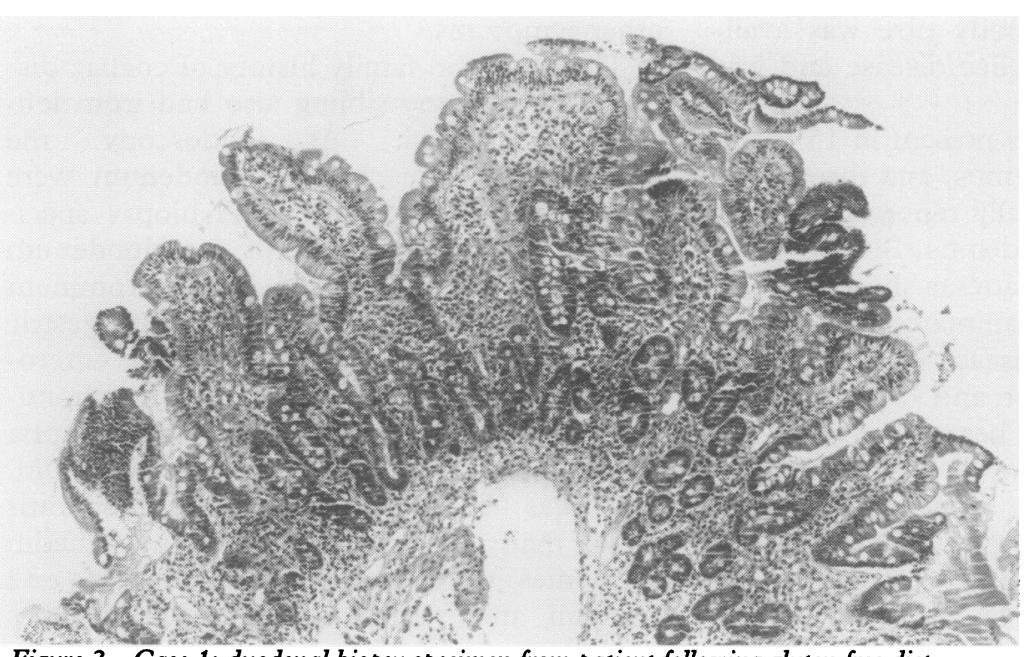

Figure 3 Case 1: duodenal biopsy specimen from patient following gluten free diet. Villous architecture is restored and surface epithelium is normal (haematoxylin and eosin).

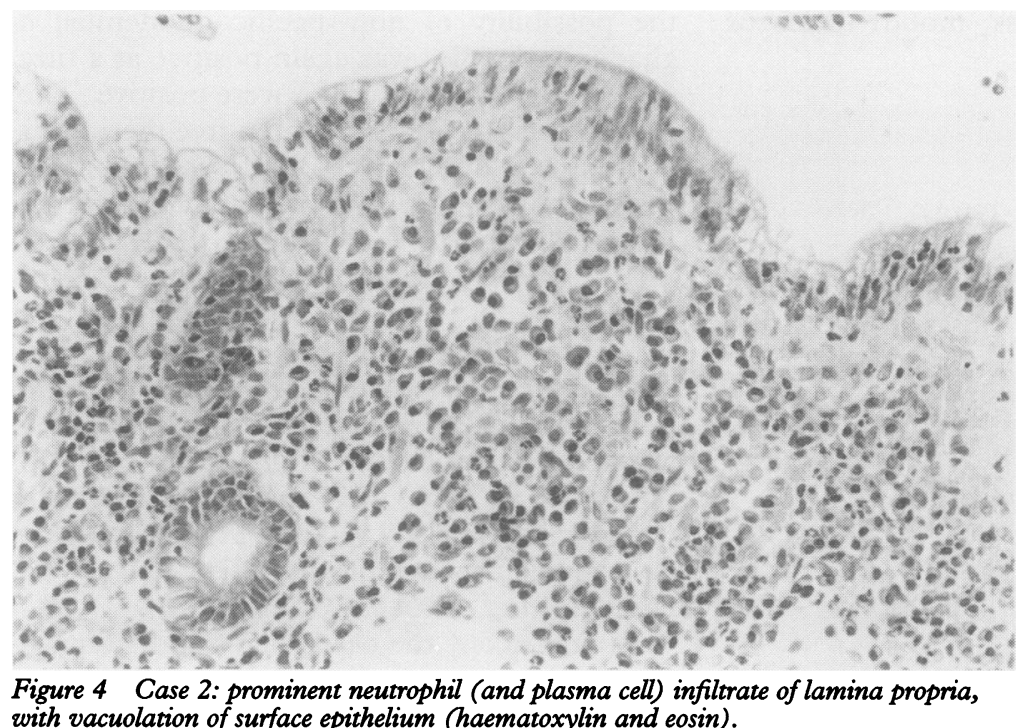

Figure 4 Case 2: prominent neutrophil (and plasma cell) infiltrate
with vacuolation of surface epithelium (haematoxylin and eosin).

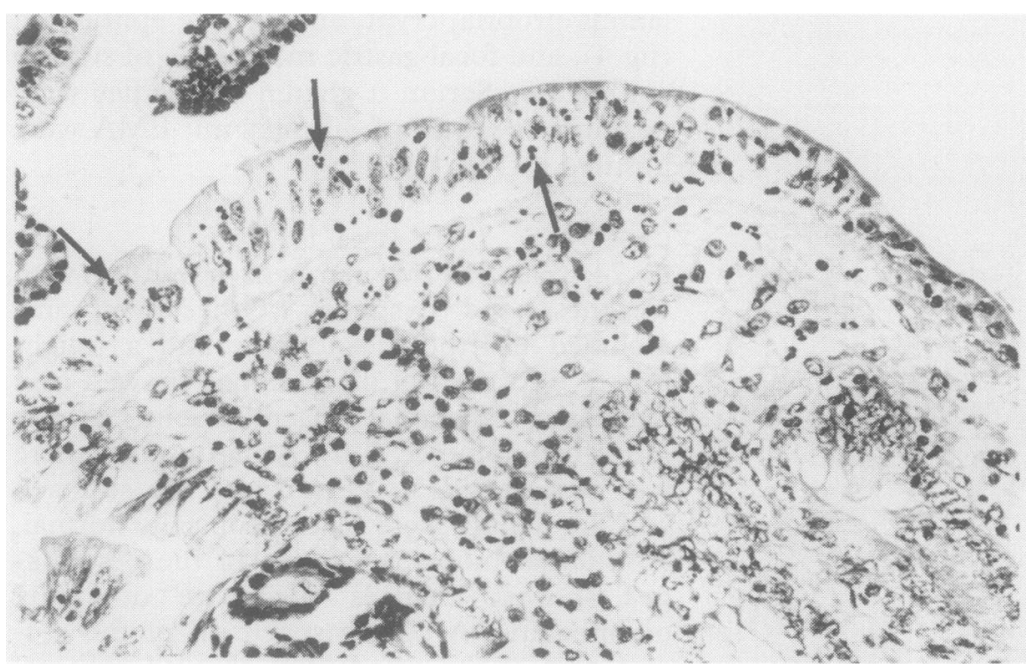

Figure 5 Case 4: duodenal surface with prominent intraepithelial polymorphonuclear leucocytes (arrows); intraepithelial lymphocytes also present (haematoxylin and eosins). lial cell abnormalities, increased numbers of intraepithelial lymphocytes and a mixed chronic inflammatory cell infiltrate of the lamina propria, with, in addition, a polymorphonuclear leucocyte infiltrate with extension upwards into the surface epithelium which also showed focal gastric metaplasia. The patient was discharged with oral iron supplements. Three months later she still had persistent abdominal discomfort. Serum $a$ gliadin antibodies were positive, at a titre of 11 units $/ \mathrm{ml}$, and she was started on a gluten free diet.

Six months later her symptoms had improved. Haemoglobin and mean cell volume were normal. At endoscopy there was mild oesophagitis. The stomach and duodenum were macroscopically normal. Duodenal biopsy specimens were normal apart from minor architectural changes and a mild chronic inflammatory cell infiltrate. Serum $a$ gliadin antibodies were positive at 10 units $/ \mathrm{ml}$.

\section{CASE 4}

A 63 year old man was anaemic (haemoglobin $99 \mathrm{~g} / \mathrm{l}$ ) on presentation for his regular donation of blood to the transfusion service. He had no family history of coeliac disease, no bowel symptoms, and no history of blood loss. As part of investigation of an iron deficiency anaemia he had a gastroscopic examination and a biopsy of the duodenum.

The mucosa was flat with one focus of gastric metaplasia (less than 5\%) and several areas with prominent polymorphonuclear leucocytes in the lamina propria, extending locally into the surface epithelium (fig 5). Intraepithelial lymphocytes were also prominent. Serum $a$ gliadin antibodies were positive, at a titre of 18 units $/ \mathrm{ml}$

As intraepithelial lymphocytes were prominent in all four cases, counts were conducted on all four, and in 11 out of the 15 cases classified as peptic ulcer $(n=3)$ or peptic duodenitis $(n=12)$. In four cases of peptic disease surface epithelium was either absent, was largely gastric, or was technically poorly preserved so that the counts were conducted

The results are as follows:

\begin{tabular}{lll}
\hline IEL/300 & $\begin{array}{l}\text { Peptic } \\
\text { ulcer disease } \\
(11 \text { cases })\end{array}$ & $\begin{array}{l}\text { Coeliac peptic } \\
\text { ulcer disease } \\
\text { (4 cases) }\end{array}$ \\
\hline surface epithelial cells & $10(5-6-$ range) & $52(43-66-$ range $)$ \\
\hline $\mathrm{p}=0.0047$ &
\end{tabular}

on the remaining 11 cases.

\section{Discussion}

With the widespread replacement of jejunal biopsy by endoscopic biopsy of the second part of the duodenum for the diagnosis of coeliac disease, histopathologists are increasingly faced with the problem of interpretation of duodenal mucosal biopsy specimens in attempting to establish the diagnosis.

The original descriptions, and most subsequent research observations of coeliac dis- 
ease, refer to histological abnormalities in jejunal mucosa. Duodenal mucosal specimens pose certain problems of interpretation in that the architecture shows shorter and thicker villi compared with those found in the jejunum, especially in areas overlying Brunner's glands. Stromal inflammation is also common and may lead to progressive shortening of villi, resulting ultimately in a flat mucosa.

Peptic duodenitis, also termed "non-specific" duodenitis, is associated with acid injury and leads to a spectrum of histological mucosal changes from mild inflammation to a flat mucosa which may be very difficult to distinguish from that seen in coeliac disease ${ }^{1}$. Grading systems for duodenitis have been proposed based on morphological changes chiefly described in the duodenal cap or first part of the duodenum (D1). Low grade duodenitis is characterised by mild widening and flattening of villi associated with mild chronic inflammation of the lamina propria. As the inflammation becomes more severe increasing flattening of architecture is noted, combined with a mixed acute and chronic inflammatory cell infiltrate of lamina propria, and with extension of polymorphonuclear leucocytes into the surface epithelium together with gastric metaplasia of the surface. ${ }^{23}$

Our four cases had flattening, gastric metaplasia, and a polymorphonuclear leucocyte exudate, but the metaplasia was never more than $5 \%$ of the surface, and the polymorphonuclear leucocytes, although prominent, were also in foci, while no erosions were seen. Some assessment of intraepithelial lymphocytes is the best way to discriminate between the two different causes of inflammation in the duodenum.

Gastric metaplasia in the duodenum has been considered a reparative or protective response to acid injury. ${ }^{4}$ Small groups of gastric type epithelial cells are commonly seen in the mucosa of $\mathrm{D} 1$, but more extensive metaplasia with extension to D2 is strongly associated with active duodenitis, low gastric $\mathrm{pH}$, and $H$ pylori infection. ${ }^{4}$ Gastric metaplasia of intestinal crypts is well recorded in chronic ulceration of the small intestine from any cause such as Crohn's disease or potassium tablets. ${ }^{6}$ Ectopic gastric mucosa has been recorded in the duodenum, jejunum, and ileum in coeliac disease, ${ }^{78}$ but gastric metaplasia of the surface epithelial cells is not generally accepted as part of the spectrum of coeliac disease. There is a strong correlation between gastric metaplasia, villous atrophy and polymorphonuclear leucocyte infiltration ${ }^{9}$ and, especially in the first part of the duodenum, polymorphonuclear leucocyte and gastric metaplasia are the strongest histological correlates of endoscopic descriptions of inflammation.

Polymorphonuclear leucocytes have not been emphasised in the descriptions of mucosal biopsy specimens in coeliac disease, although they seem to have some role in the pathogenesis of mucosal injury in coeliac disease as the early response to gluten challenge in both jejunal ${ }^{10}$ and rectal $^{11}$ mucosa is characterised by an acute inflammatory response in the lamina propria with vascular dilatation, prominent polymorphonuclear leucocyte exudation, and mast cell degranulation. Secretion of neutrophil enzymes (such as myeloperoxidase) into jejunal juice has also been shown to be increased following gluten challenge. ${ }^{12}$ The early mucosal acute inflammatory response to gluten challenge is followed by increasing lymphocytic infiltration of the lamina propria, migration of lymphocytes into the surface epithelium, and reduction in the polymorphonuclear leucocyte population. A substantial increase in polymorphonuclear leucocytes has been reported in jejunal mucosal biopsy specimens of patients with untreated coeliac disease using ultrathin sections stained with toluidine blue, ${ }^{13}$ but in $4 \mu \mathrm{m}$ paraffin wax embedded routine sections stained with haematoxylin and eosin polymorphonuclear leucocytes are generally referred to as scanty or absent. ${ }^{14}$

Although substantial polymorphonuclear leucocyte infiltrate and surface epithelial gastric metaplasia have been recommended as pointers strongly favouring peptic duodenitis over coeliac disease, ${ }^{14}$ they are not specific to acid induced injury and should not therefore be used as criteria to exclude the diagnosis of coeliac disease. We have shown here that small foci of gastric epithelium and focal exudates of polymorphonuclear leucocytes may be found in coeliac disease, and that assessment of intraepithelial lymphocytes is a reliable morphological discriminant to distinguish between coeliac disease and peptic duodenitis. It has been suggested that peptic duodenitis and coeliac disease may coexist in the same patient ${ }^{15}$ and this could be invoked to explain the changes in the cases we report here. The symptomatic and morphological improvement seen in our patients following gluten withdrawal without any treatment aimed at the acid-peptic disease implies that the structural changes were in fact due to coeliac disease.

In patients with clinical features suggestive of coeliac disease, morphological abnormalities of duodenal mucosa should be interpreted in conjunction with the clinical information with particular reference to dietary exclusion of gluten and immunological confirmation using $\alpha$ gliadin and endomysial antibody titres. Definitive diagnosis in cases where morphology is not typical depends on consideration of all of these modalities rather than a reliance on individual morphological structural criteria.

We thank Ms Orla Shiels for photographic assistance and Ms Truda McCullagh for typing the manuscript.

1 Dobbins WO. Diagnostic pathology of the intestinal mucosa. New York: Springer-Verlag, 1990

2 Whitehead R, Roca M, Meikle DD, Skinner J, Truelove SC. The histological classification of duodenitis in fibreoptic biopsy specimens. Digestion 1975;13:129-31.

3 McCallum R W, Singh D, Wollam J. Endoscopic and histologic correlations of the duodenal bulb. Arch Pathol Lab Med 1979;103:169-72.

4 Wyatt SS, Rathbone BJ, Dixon MF, Heatley RV. 
Campylobacter pyloridis and acid induced gastric metaplasia in the pathogenesis of duodenitis. $\mathscr{f}$ Clin Pathol 1987;40:841-8.

5 Wyatt JL, Rathbone BJ, Sobala GM, et al. Gastric epithelium in the duodenum: its association with Helicobacter pylori and inflammation. $f$ Clin Pathol 1990;43:981-6.

6 Bayless TM, Kapelowitz RF, Shelley WM, Ballinger WF, Hendrix TR. Intestinal ulceration, a complication of coeliac disease. N Engl f Med 1967;276:996-1002.

7 Trier JS, Moxey PC, Fordtran JS, MacDermott RP. Ectopic gastric mucosa in coeliac sprue. Gastroenterology 1973;65:712-17.

8 Thompson H. Necropsy studies on adult coeliac disease. 7 Clin Pathol 1974;27:710-21.

9 Jenkins D, Goodall A, Gillet FR, Scott BB. Defining duodenitis: quantitative histological study of mucosal responses and their correlations. $f$ Clin Pathol 1985;38: 1119-26.

10 Anand BS, Piris J, Jerrome DW, Offord RE, Truelove SC. The timing of histological damage following a single challenge with gluten in treated coeliac disease. $Q \mathcal{F} \mathrm{Med}$ 1981;197:83-94.

11 Loft DE Marsh MN, Sandle GI, et al Studies of intestinal lymphoid tissue XII. Epithelial lymphocyte and mucosal responses to rectal gluten challenge in celiac mucosal responses to rectal gluten chall

12 Hallgren R, Colambel JF, Dahl R, et al. Neutrophil and eosinophil involvement of the small bowel in patien with celiac disease and Crohn's disease. $\mathrm{Am} f \mathrm{Med}$ 1989:86;56-64.

13 Dheshi I, Marsh M N, Kelly C, Crowe P. Morphometric analysis of small intestinal mucosa. (II). Determination of lamina propria volumes; plasma cell and neutrophil populations within control and coeliac disease mucosae. Virchows Arch (Pathol Anat) 1984;403:173-80.

14 Day DW, Husain OAN. Biopsy pathology of the oesophagus, stomach and duodenum. London: Chapman and Hall, 1986.

15 Bayless TM, Jones B, Hamilton SR, Yardley JH. Nodular duodenal bulb and peptic duodenitis is incompletely responsive "atypical" sprue. Gastroenterology 1982;82: 1014 